\title{
A MINIMUM ENTROPY IMAGE DENOISING ALGORITHM Minimizing Conditional Entropy in a New Adaptive Weighted K-th Nearest Neighbor Framework for Image Denoising
}

\author{
Cesario Vincenzo Angelino, Eric Debreuve, Michel Barlaud \\ Laboratory I3S University of Nice-Sophia Antipolis/CNRS, 2000, route des Lucioles - 06903 Sophia Antipolis, France \\ \{angelino, debreuve, barlaud\}@i3s.unice.fr
}

Keywords: Image denoising,variational methods, entropy, k-th nearest neighbors.

\begin{abstract}
In this paper we address the image restoration problem in the variational framework. The focus is set on denoising applications. Natural image statistics are consistent with a Markov random field (MRF) model for the image structure. Thus in a restoration process attention must be paid to the spatial correlation between adjacent pixels. The proposed approach minimizes the conditional entropy of a pixel knowing its neighborhood. The estimation procedure of statistical properties of the image is carried out in a new adaptive weighted k-th nearest neighbor (AWkNN) framework. Experimental results show the interest of such an approach. Restoration quality is evaluated by means of the RMSE measure and the SSIM index, more adapted to the human visual system.
\end{abstract}

\section{INTRODUCTION}

The goal of image restoration is to recover an image that has been degraded by some stochastic process. Research focus was set on removing additive, independent, random noise. However, more general degradation phenomenons can be modeled, such as blurring, non-independent noise, and so on. When the only degradation present in an image is noise, the model is described by the equation

$$
\tilde{X}=X+N
$$

where $\tilde{X}$ is the degraded image, $X$ is the ideal image and $N$ is the additive noise term.

The literature in image restoration is vast and several techniques have been proposed in different frameworks such as linear and nonlinear filtering in either the spatial or a transform domain. Nonlinear filtering approaches are typically based on variational methods, leading to algorithm based on partial differential equations. Indeed, these methods define an energy functional based on geometric or statistical properties of images. The minimization of this functional leads to the correspondent partial differential equations or evolution equation. A great deal of image processing work developments are based on a stochastic model of image structure given by Markov random fields (MRFs) (Geman and Geman, 1990). Recent researches (Huang and Mumford, 1999; Lee et al., 2003; Carlsson et al., 2007) confirm that the statistics of natural images are consistent with the MRF model for the image structure, showing that a spatial correlation between adjacent pixel exists. This correlation must be take into account in the restoration process to preserve the image structures. The idea is to consider the intensity of a pixel jointly with the intensities of its neighborhood. In particular the conditional entropy of the pixel intensity knowing its neighborhood is minimized (Awate and Whitaker, 2006). We use entropy because it provides a measure of dispersion of the random variable (Cover and Thomas, 1991) and it is robust to the presence of outliers in the samples. The underlying PDF can be estimated directly from the data using a common nonparametric Parzen windowing method. However Parzen methods presents some drawbacks that can be avoided using the k-th nearest neighbor $(\mathrm{kNN})$ methods, as shown in section 5. The experimental results show the slightly better performance of the latter method with respect to Parzen methods. The quality of restored images is evaluated by the largely used RMSE measure and also by means of the Structure Similarity (SSIM) measure 
(Wang et al., 2004), more adapted to the human visual system (HVS).

This paper is organized as follows. In section 2 the energy and its derivative are presented and the adaptive weighted kNN framework is introduced. In section 3 a high level overview of the algorithm is given and in section 4 some experimental results are shown. Finally, discussion and future works are proposed in the last section.

\section{KNN RESTORATION}

\subsection{Image Model}

Let us model the images as random field. A random field is a family of random variables $X(\Omega ; T)$ for some index set $T$, where, for each fixed $T=t$, the random variable is defined on the sample space $\Omega$. If we fix $\Omega=\omega$ and let $T$ be a set of points defined on a discrete Cartesian grid, we have a realization of the random field called the digital image, $X(\omega, T)$. In this case, $\{t\}_{t \in T}$ is the set of pixels in the image. For twodimensional images, $t$ is a two-vector. If we fix $T=t$, and let $\omega$ vary, then $X(t)$ is a random variable on the sample space. We denote a specific realization $X(\omega ; t)$ (the intensity at pixel $t$ ) a deterministic function $x(t)$. If we associate with $T$ a family of pixel neighborhoods $N=\left\{N_{t}\right\}_{t \in T}$ such that $N_{t} \subset T, t \notin N_{t}$, and $u \in N_{t}$ if and only if $t \in N_{u}$, then $N$ is called a neighborhood system for the set $T$. Points in $N_{t}$ are called neighbors of $t$. We define a random vector $Y(t)=$ $\{X(t)\}_{t \in N_{t}}$, corresponding to the set of intensities at the neighbors of pixel $t$. We also define a random vector $Z(t)=(X(t), Y(t))$ to denote image regions, i.e., pixels combined with their neighborhoods.

\subsection{Energy and Derivative}

Image restoration is an inverse problem, that can be formulated as a functional minimization problem. We consider the conditional entropy functional, i.e., the uncertainty of the random pixel $X$ when its neighborhood is given, as suitable measure for denoising applications (Awate and Whitaker, 2006). Thus the recovered image satisfies

$$
X^{*}=\arg \min _{\mathrm{X}} \mathrm{h}\left(\tilde{\mathrm{X}} \mid \tilde{\mathrm{Y}}=\mathrm{y}_{\mathrm{i}}\right)
$$

Entropy functional can be approximated by the following estimator (Ahmad and Lin, 1976)

$$
h\left(X \mid Y=y_{i}\right) \approx-\frac{1}{|T|} \sum_{t_{j} \in T} \log p\left(x_{j} \mid y_{i}\right),
$$

where

$$
p\left(s \mid y_{i}\right)=\frac{1}{\left|T_{y_{i}}\right|} \sum_{t_{m} \in T_{y_{i}}} K_{h}\left(s-x_{m}\right),
$$

is the Parzen kernel estimate of the PDF. $T_{y_{i}}$ is the set of index pixels which have the same neighborhood $y_{i}$. In order to solve the optimization problem (2) a steepest descent algorithm is used. The energy derivative of (3) is (see Appendix for the demonstration)

$$
\frac{\partial h\left(X \mid Y=y_{i}\right)}{\partial x_{i}}=-\frac{1}{|T|} \frac{\nabla p\left(z_{i}\right)}{p\left(z_{i}\right)} \frac{\partial z_{i}}{\partial x_{i}}+\chi\left(x_{i}\right),
$$

with

$$
\chi\left(x_{i}\right)=\frac{1}{|T|} \frac{1}{\left|T_{y_{i}}\right|} \sum_{t_{j} \in T} \frac{\nabla K_{h}\left(x_{j}-x_{i}\right)}{p\left(x_{j} \mid y_{i}\right)} .
$$

The term $\chi(\cdot)$ in (6) is difficult to estimate. However if the Parzen kernel $K_{h}(\cdot)$ has a narrow window size, only samples very close to the actual estimation point will contribute to the pdf. Under this assumption the conditional pdf is $p\left(s \mid y_{i}\right) \approx N_{s} /\left|T_{y_{i}}\right|$, where $N_{s}$ is the number of pixels equal to $s$. Thus by substituting and observing that $\nabla K_{h}(\cdot)$ is an odd function, we observe that $\chi\left(x_{i}\right)$ is negligible for almost every value assumed by $x_{i}$. In the following we will not consider this term. Thus the energy derivative is a mean-shift (Comaniciu and Meer, 2002) term on the high dimensional joint pdf multiplied by a projection term. The mean-shift is a simple estimate (Fukunaga and Hostetler, 1975) of $\nabla \log p\left(z_{i}\right)$.

$$
\frac{\nabla p\left(z_{i}\right)}{p\left(z_{i}\right)}=\frac{d+2}{h^{2}} \frac{1}{k\left(z_{i}, h\right)} \sum_{z_{j} \in S_{h}\left(z_{i}\right)} K_{h}\left(z_{j}-z_{i}\right),
$$

where $d$ is the dimension of $Z, S_{h}\left(z_{i}\right)$ is the support of the Parzen kernel centered at point $z_{i}$ and of size $h, k$ being the number of observation falling into $S_{h}(X)$.

\subsection{Limitations of Parzen Windowing}

The Parzen method makes no assumption about the actual PDF and is therefore qualified as nonparametric. The choice of the kernel window size $h$ is critical (Scott, 1992). If $h$ is too large, the estimate will suffer from too little resolution, otherwise if $h$ is too small, the estimate will suffer from too much statistical variability.

As the dimension of the data space increases, the space sampling gets sparser (problem known as the curse of dimensionality). Therefore, less samples fall into the Parzen window centered on each sample, making the PDF estimation less reliable. Dilating the Parzen window does not solve this problem since it 
leads to over-smoothing the PDF. In a way, the limitations of the Parzen Method come from the fixed window size: the method cannot adapt to the local sample density. The $k$-th nearest neighbor $(\mathrm{kNN})$ framework provides an advantageous alternative.

\section{$2.4 \quad K$-th Nearest Neighbor Method}

In the Parzen-window approach, the PDF at sample $s$ is related to the number of samples falling into a window of fixed size centered on the sample. The $\mathrm{kNN}$ method is the dual approach: the density is related to the size of the window necessary to include the $k$ nearest neighbors of the sample. The choice of $k$ appears to be much less critical than the choice of $h$ in the Parzen method. In the kNN framework, the mean-shift vector is given by (Fukunaga and Hostetler, 1975)

$$
\frac{\nabla p\left(z_{i}\right)}{p\left(z_{i}\right)}=\frac{d+2}{\rho_{k}^{2}}\left[\left(\frac{1}{k} \sum_{z_{j} \in S_{\rho_{k}}} z_{j}\right)-z_{i}\right],
$$

where $\rho_{k}$ is the distance to the $k$-th nearest neighbor.

\subsection{Adaptive Weighted kNN Approach}

The kNN method provides several advantages with respect to the Parzen Window method such as the number of samples falling in the window is fixed and known. Thus even if the space sampling gets sparser, we cannot have windows with zero samples falling into them. Moreover, the window size is locally adaptive. However, near the distribution modes there is a high density of samples. The window size associated to the $k$-th nearest neighbor could be too small. In this case the estimate will be sensible to the statistical variation in the distribution. To avoid this problem we would increase the number of nearest neighbors, to have an appropriate window size near the modes. However this choice would produce in the tails of the distribution a window too large. Thus very far samples would contribute to the estimation.

We propose, as an alternative solution, to weight the contribution of the samples, i.e.,

$$
\frac{1}{k} \sum_{z_{j} \in S_{\rho_{k}}} z_{j} \rightarrow \frac{1}{\sum_{j=1}^{k} w_{j}} \sum_{\substack{j=1 \\ z_{j} \in S_{\rho_{k}}}}^{k} w_{j} z_{j} .
$$

Intuitively, the weights must be a function of distance between the actual sample and the $j$-th nearest neighbor, i.e., samples with smaller distance are weighted more heavily than ones with larger distance. Several weight functions (WFs) may be considered. For instance, a simple function is

$$
w_{j}=1-\left(\frac{\rho_{j}}{\rho_{k}}\right)^{p} \text { with } p \in[0,+\infty[,
$$

where $\rho_{j}$ is the distance to the actual sample of the $j$ th nearest neighbor and $\rho_{k}$ indicate the distance of the farthest ( $k$-th) neighbor. For $p=1$, eq.(10) is equivalent to the linear WF proposed by Dudani (Dudani, 1976) and for $p=2$, we have a quadratic WF. Drawbacks of this simple WF are the zero-value at boundaries and the function behavior fixed by the $\rho_{k}$ value. Let us consider now a gaussian WF

$$
w_{j}=\exp -\frac{\left(\rho_{j} / \rho_{k}\right)^{2}}{\alpha} .
$$

In particular this WF is equivalent to apply a gaussian mask of variance $\alpha / 2$ in the actual window of size $\rho_{k}$. As $\max _{j} \rho_{j} / \rho_{k}=1$, the weights belong to $[\exp -1 / \alpha, 1]$. Thus an appropriate value of $\alpha$ must be chosen (see Fig.1). We would have a quite uniform weighting process (large gaussian variance) when $\rho_{k}$ is not quite large (i.e., near the distribution modes), and a smaller variance in the distribution tails $\left(\rho_{k}\right.$ large) to reduce the effective window size. Thus a single value for $\alpha$ is clearly inappropriate.

We propose an adaptive value for $\alpha$ to locally match the distribution

$$
\alpha=\frac{1}{8}\left(\frac{\rho_{k}^{\max }}{\rho_{k}}\right)^{2}
$$

and the corresponding WF is

$$
w_{j}=\exp -\frac{1}{8}\left(\frac{\rho_{j}}{\rho_{k}^{\max }}\right)^{2} .
$$

Thus the mean shift term in (8) is replaced by

$$
\frac{\nabla p\left(z_{i}\right)}{p\left(z_{i}\right)}=\frac{d+2}{\rho_{k}^{2}} M_{k}\left(z_{i}\right)
$$

where

$$
M_{k}\left(z_{i}\right)=\left(\frac{1}{\sum_{j=1}^{k} w_{j}} \sum_{\substack{j=1 \\ z_{j} \in S_{\rho_{k}}}} w_{j} z_{j}\right)-z_{i}
$$

and $w_{j}$ given by eq.(13).

\section{ALGORITHM OVERVIEW}

The method proposed minimizes the conditional entropy (3) using a gradient descent. The derivative (5) is estimated in the adaptive weighted $\mathrm{kNN}$ framework, as explained in section 2.5. In this case the term 


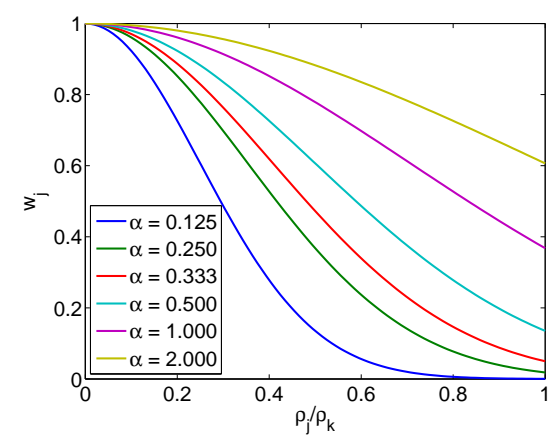

Figure 1: Weight function behavior as function of $\alpha$.

$\nabla p / p$ is expressed by eq.(14). Thus the steepest descent algorithm is performed with the following evolution equation

$$
x_{i}^{(n+1)}=x_{i}^{(n)}-v \frac{d+2}{\rho_{k}^{2}} M_{k}\left(z_{i}\right) \frac{\partial z_{i}}{\partial x_{i}},
$$

where $v$ is the step size.

At each iteration the mean-shift vector (15) in the high dimensional space $Z$ is calculated. The high dimensional space is given by considering jointly the intensity of the current pixel and that of its neighborhood, as explained in section 2.1. The pixel value $x_{i}$ is then updated by means of eq.(16).

The $k$ nearest neighbors are provided by the Approximate Nearest Neighbor Searching (ANN) library (Mount and Arya, ). Indeed computing exact nearest neighbors in dimensions much higher than 8 seems to be a very difficult task. Few methods seem to be significantly better than a brute-force computation of all distances. However, it has been shown that by computing nearest neighbors approximately, it is possible to achieve significantly faster running times often with a relatively small actual errors.

\section{EXPERIMENTAL RESULTS}

In this section, some results from the algorithm proposed are shown. In order to measure the performance of our algorithm we degraded the Lena image (256x256 pixel) by adding a Gaussian noise with standard deviation $\sigma=10$. We consider a 9 x 9 neighborhoods, and we add spatial features to the original radiometric data (Elgammal et al., 2003; Boltz et al., 2007). Formally, the PDF of $Z(t), t \in T$, is replaced with the PDF of $\{Z(t), t\}, t \in T$. These spatial features allow us to reduce the effect of the non stationarity of the signal in the estimation process, by preferring regions closer to the estimation point. The dimension of the data $d$ is therefore equal to 83 , and we have to search the $k$ nearest neighbors in such a high
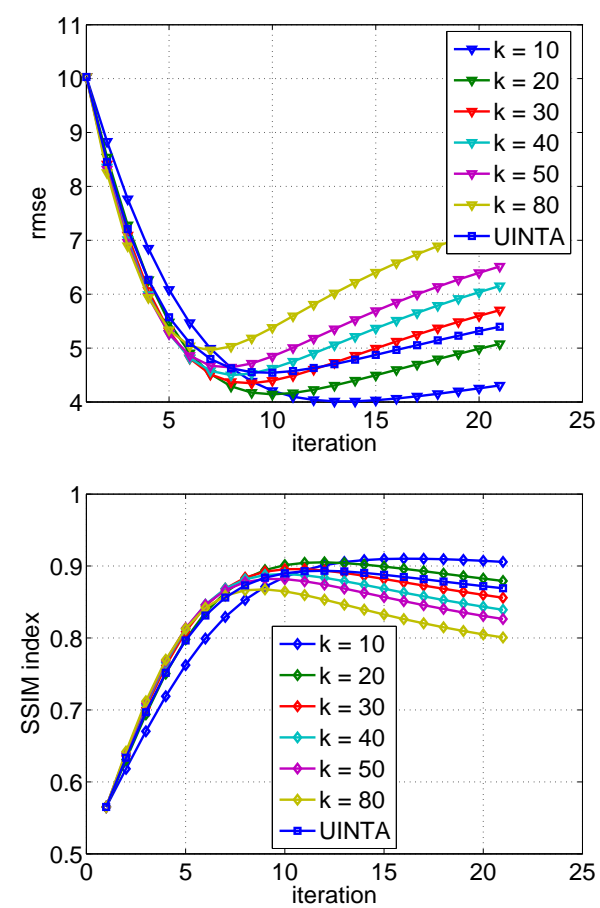

Figure 2: RMSE and SSIM in function of algorithm iterations for different values of $k$.

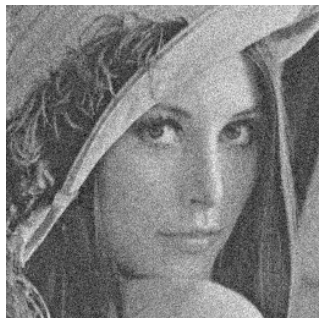

a)

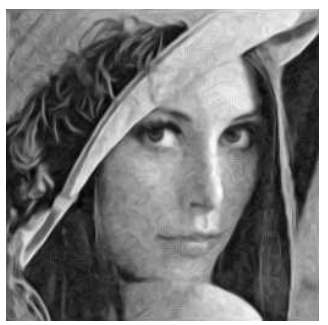

c)

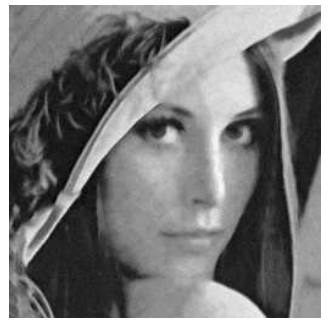

b)

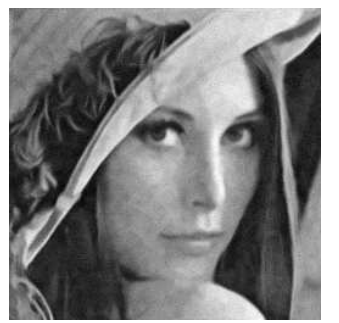

d)
Figure 3: Comparison of restored images. (a) Noisy image. (b) UINTA restored. (c) $\mathrm{kNN}$ restored, $\mathrm{k}=10$. (d) $\mathrm{kNN}$ restored, $\mathrm{k}=40$.

dimensional space. Let us remind that the search is performed using the ANN library. The algorithm performs a gradient descent as described in section 3 . Figure 3 shows a comparison of the restored images. Fig. 2 shows the RMSE and SSIM curves as a function of the algorithm iterations, for the UINTA (Awate 
and Whitaker, 2006) algorithm, and our kNN based restoration method with different values of $k$. In Table1, the optimal values of the RMSE and SSIM are shown. Our algorithm provides results comparable with UINTA and slightly better when $k \in[10 ; 50]$. However a small value of $k$ produces some artifacts in the restored images, as shown in Fig.4. A larger value of $k$ results in an increasing processing time. Moreover the restored images seem to lost some details, for instance, on the hat. An intermediate value of $k=40$ is a good compromise between the quality of the restored image and the processing time. In terms of speed, our algorithm is much faster than UINTA, due to the adaptive weighted kNN framework. Indeed, UINTA have to update the Parzen window size at each iteration. To do this a cross validation optimization is performed. On the contrary our method simply adapts the PDF changes during the minimization process. For instance, cpu time in the Matlab environment for standard UINTA algorithm is almost $4500 \mathrm{sec}$. Our algorithm, with $k=10$, only requires around $600 \mathrm{sec}$.

\begin{tabular}{|c|c|c|}
\hline$k$ & RMSE & SSIM \\
\hline 3 & 5.511 & 0.918 \\
\hline 5 & 4.219 & 0.917 \\
\hline 10 & 4.012 & 0.910 \\
\hline 15 & 4.061 & 0.907 \\
\hline 20 & 4.142 & 0.905 \\
\hline 30 & 4.347 & 0.895 \\
\hline 40 & 4.498 & 0.889 \\
\hline 50 & 4.642 & 0.882 \\
\hline 80 & 4.960 & 0.867 \\
\hline 100 & 5.117 & 0.832 \\
\hline 200 & 5.541 & 0.805 \\
\hline UINTA & 4.651 & 0.890 \\
\hline
\end{tabular}

Table 1: RMSE and SSIM values for different values of $k$, and for UINTA.

\section{CONCLUSION}

This paper presents a restoration method in the variational framework based on the minimization of the conditional entropy using the kNN framework. In particular a new adapted weighted kNN (AWkNN) approach has been proposed. The simulations indicated slightly better results in RMSE and SSIM measures w.r.t. the UINTA algorithm and a marked gain in cpu speed. This gain is due to the property of AWkNN that simply adapts the PDF changes during the minimization process. Results are even more promising considering that no regularization is applied. As future works, a regularization method will be taken into account. Moreover, the feature space dimension can be boosted by taking into account other image related features. For instance, the image gradient components can be used as additional features.

\section{REFERENCES}

Ahmad, I. A. and Lin, P. (1976). A nonparametric estimation of the entropy for absolutely continuous distributions. IEEE TRANSACTIONS ON INFORMATION THEORY.

Awate, S. P. and Whitaker, R. T. (2006). Unsupervised, information-theoretic, adaptive image filtering for image restoration. IEEE Trans. Pattern Anal. Mach. Intell., 28(3):364-376.

Boltz, S., Debreuve, E., and Barlaud, M. (2007). Highdimensional statistical distance for region-of-interest tracking: Application to combining a soft geometric constraint with radiometry. In IEEE International Conference on Computer Vision and Pattern Recognition, Minneapolis, USA. CVPR'07.

Carlsson, G., Ishkhanov, T., de Silva, V., and Zomorodian, A. (2007). On the local behavior of spaces of natural images. International Journal of Computer Vision.

Comaniciu, D. and Meer, P. (MAY, 2002). Mean shift: A robust approach toward feature space analysis. IEEE TRANSACTIONS ON PATTERN ANALYSIS AND MACHINE INTELLIGENCE, 24, NO.5:603-619.

Cover, T. and Thomas, J. (1991). Elements of Information Theory. Wiley-Interscience.

Dudani, S. (1976). The distance-weighted k-nearestneighbor rule. 6(4):325-327.

Elgammal, A., Duraiswami, R., and Davis, L. S. (2003). Probabilistic tracking in joint feature-spatial spaces. pages 781-788, Madison, WI.

Fukunaga, K. and Hostetler, L. D. (JANUARY, 1975). The estimation of the gradient of a density function, with applications in pattern recognition. IEEE TRANSACTIONS ON INFORMATION THEORY, 21, NO.1:3240.

Geman, S. and Geman, D. (1990). Stochastic relaxation, gibbs distributions, and the bayesian restoration of images. pages 452-472.

Huang, J. and Mumford, D. (1999). Statistics of natural images and models. pages 541-547.

Lee, A. B., Pedersen, K. S., and Mumford, D. (2003). The nonlinear statistics of high-contrast patches in natural images. Int. J. Comput. Vision, 54(1-3):83-103.

Mount, D. M. and Arya, S. Ann: A library for approximate nearest neighbor searching. http://www.cs.umd.edu/ mount/ANN/.

Scott, D. (1992). Multivariate Density Estimation: Theory, Practice, and Visualization. Wiley.

Wang, Z., Bovik, A. C., Sheikh, H. R., and Simoncelli, E. P. (APRIL, 2004). Image quality assessment: From error 


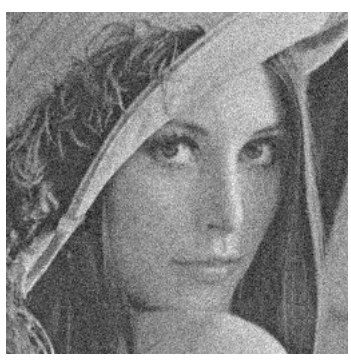

a) Degraded image

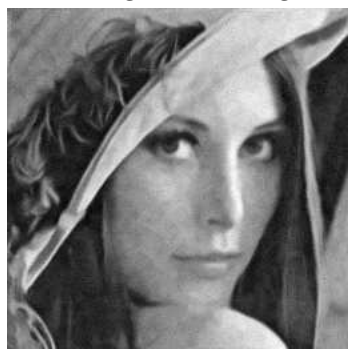

e) $k=40$

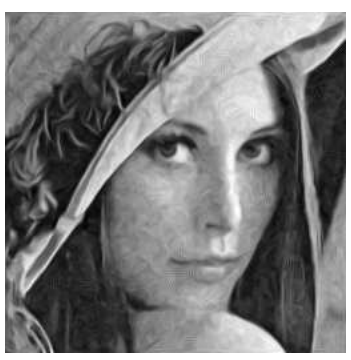

b) $k=10$

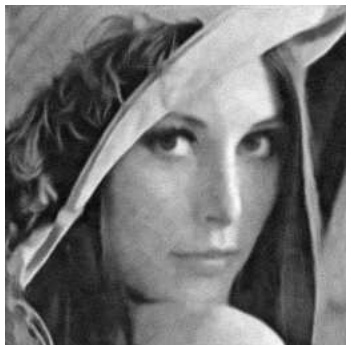

f) $k=50$

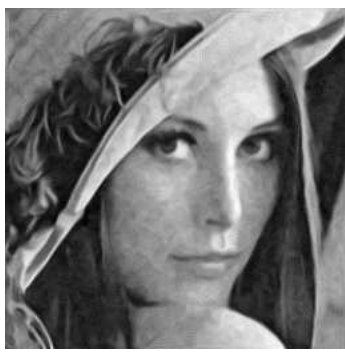

c) $k=20$

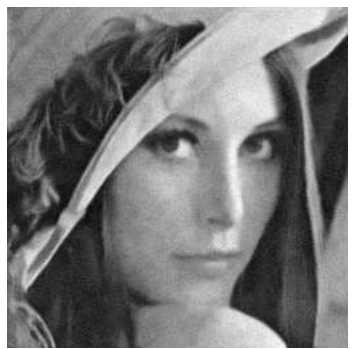

g) $k=80$

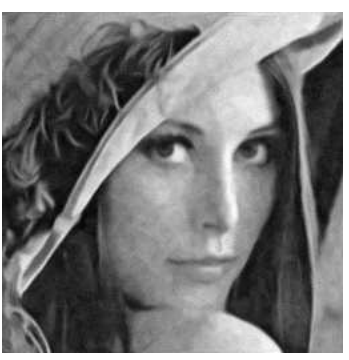

d) $k=30$

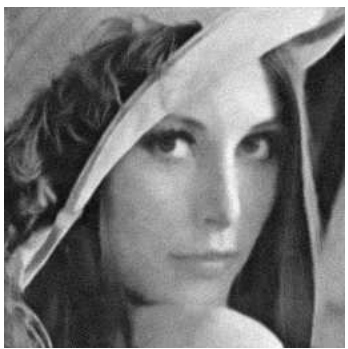

h) $k=100$

Figure 4: Comparison of knn restored images with different values of $k$.

visibility to structural similarity. IEEE TRANSACTIONS ON IMAGE PROCESSING, 13, NO.4:600612.

\section{APPENDIX}

In this section the calculus of the derivative of the conditional entropy of eq.(3) is performed. Let us remind that

$$
h\left(X \mid Y=y_{i}\right) \approx-\frac{1}{|T|} \sum_{t_{j} \in T} \log p\left(x_{j} \mid y_{i}\right)
$$

and

$$
p\left(s \mid y_{i}\right)=\frac{1}{\left|T_{y_{i}}\right|} \sum_{t_{m} \in T_{y_{i}}} K\left(s-x_{m}\right),
$$

where $T_{y_{i}}$ is the set of index pixels which have the same neighborhood $y_{i}$. Thus we have

$h\left(X \mid Y=y_{i}\right)=-\frac{1}{|T|} \sum_{t_{j} \in T} \log \left[\frac{1}{\left|T_{y_{i}}\right|} \sum_{t_{m} \in T_{y_{i}}} K\left(x_{j}-x_{m}\right)\right]$.

and, by taking the derivative of (19),

$\frac{\partial h\left(X \mid Y=y_{i}\right)}{\partial x_{i}}=-\frac{1}{T} \sum_{t_{j} \in T} \frac{1}{p\left(x_{j} \mid y_{i}\right)} \frac{1}{T_{y_{i}}} \sum_{t_{m} \in T_{y_{i}}} \frac{\partial K\left(x_{j}-x_{m}\right)}{\partial x_{i}}$.

The last term in (20) is equal to

$$
\frac{\partial K\left(x_{j}-x_{m}\right)}{\partial x_{i}}=\left\{\begin{array}{cc}
-\nabla K\left(x_{j}-x_{m}\right) \delta_{m-i} & j \neq i \\
\left(1-\delta_{m-i}\right) \nabla K\left(x_{j}-x_{m}\right) & j=i
\end{array}\right.
$$

Thus by substituting

$$
\begin{aligned}
\frac{\partial h\left(X \mid Y=y_{i}\right)}{\partial x_{i}} & =-\frac{1}{|T|} \frac{\nabla p\left(x_{i} \mid y_{i}\right)}{p\left(x_{i} \mid y_{i}\right)} \\
& +\frac{1}{|T|} \frac{1}{\left|T_{y_{i}}\right|} \sum_{t_{j} \in T} \frac{\nabla K\left(x_{j}-x_{i}\right)}{p\left(x_{j} \mid y_{i}\right)} .
\end{aligned}
$$

By multiplying the numerator and denominator of the first term in (22) with $p\left(y_{i}\right)$

$$
\frac{\nabla p\left(x_{i} \mid y_{i}\right)}{p\left(x_{i} \mid y_{i}\right)} \cdot \frac{p\left(y_{i}\right)}{p\left(y_{i}\right)}=\frac{\nabla p\left(z_{i}\right)}{p\left(z_{i}\right)} \cdot \frac{\partial z_{i}}{\partial x_{i}},
$$

where the projection operator $\partial z_{i} / \partial x_{i}$ is because we change from $x_{i}$ to $z_{i}$. Finally, the derivative of (3) is

$$
\begin{aligned}
\frac{\partial h\left(X \mid Y=y_{i}\right)}{\partial x_{i}}= & -\frac{1}{|T|} \frac{\nabla p\left(z_{i}\right)}{p\left(z_{i}\right)} \frac{\partial z_{i}}{\partial x_{i}} \\
& +\frac{1}{|T|} \frac{1}{\left|T_{y_{i}}\right|} \sum_{t_{j} \in T} \frac{\nabla K\left(x_{j}-x_{i}\right)}{p\left(x_{j} \mid y_{i}\right)} .
\end{aligned}
$$

The second additional term can be neglected under certain hypothesis. Thus the energy derivative is a mean-shift (Comaniciu and Meer, 2002) term on the high dimensional joint pdf multiplied by a projection term. 